\title{
Vertebral fractures among breast cancer survivors in China: a cross-sectional study of prevalence and health services gaps
}

Evelyn Hsieh ${ }^{1}$, Qin Wang ${ }^{2}$, Renzhi Zhang ${ }^{3}$, Xin Niư ${ }^{4}$, Weibo Xia ${ }^{5}$, Liana Fraenkel ${ }^{1}$, Karl L. Insogna ${ }^{6}$, Jing Li ${ }^{3}$, Jennifer S. Smith ${ }^{7}$, Chunwu Zhou ${ }^{3}$, You-lin Qiao ${ }^{4}$ and Pin Zhang ${ }^{8^{*}}$

\begin{abstract}
Background: Breast cancer survivors are at high risk for fracture due to cancer treatment-induced bone loss, however, data is scarce regarding the scope of this problem from an epidemiologic and health services perspective among Chinese women with breast cancer.

Methods: We designed a cross-sectional study comparing prevalence of vertebral fractures among age- and BMImatched women from two cohorts. Women in the Breast Cancer Survivors cohort were enrolled from a large cancer hospital in Beijing. Eligibility criteria included age 50-70 years, initiation of treatment for breast cancer at least 5 years prior to enrollment, and no history of metabolic bone disease or bone metastases. Data collected included sociodemographic characteristics; fracture-related risk factors, screening and preventive measures; breast cancer history; and thoracolumbar $x$-ray. The matched comparator group was selected from participants enrolled in the Peking Vertebral Fracture Study, an independent cohort of healthy community-dwelling postmenopausal women from Beijing.

Results: Two hundred breast cancer survivors were enrolled (mean age $57.5 \pm 4.9$ years), and compared with 200 matched healthy women. Twenty-two (11\%) vertebral fractures were identified among breast cancer survivors compared with 7 (3.5\%) vertebral fractures in the comparison group, yielding an adjusted odds ratio for vertebral fracture of $4.16(95 \% \mathrm{Cl} 1.69-10.21, p<0.01)$. The majority had early stage (85.3\%) and estrogen and/or progesterone receptor positive (84.6\%) breast cancer. Approximately half of breast cancer survivors reported taking calcium supplements, $6.1 \%$ reported taking vitamin D supplements, and only $27 \%$ reported having a bone density scan since being diagnosed with breast cancer.

Conclusions: Despite a four-fold increased odds of prevalent vertebral fracture among Chinese breast cancer survivors in our study, rates of screening for osteoporosis and fracture risk were low reflecting a lack of standardization of care regarding cancer-treatment induced bone loss.
\end{abstract}

Keywords: Breast cancer, Cancer treatment-induced bone loss, Vertebral fracture, China

\footnotetext{
* Correspondence: zppumc@163.com

${ }^{8}$ Department of Medical Oncology, National Cancer Center/Cancer Hospital,

Chinese Academy of Medical Sciences \& Peking Union Medical College,

Beijing, China

Full list of author information is available at the end of the article
}

(c) The Author(s). 2018 Open Access This article is distributed under the terms of the Creative Commons Attribution 4.0 International License (http://creativecommons.org/licenses/by/4.0/), which permits unrestricted use, distribution, and reproduction in any medium, provided you give appropriate credit to the original author(s) and the source, provide a link to the Creative Commons license, and indicate if changes were made. The Creative Commons Public Domain Dedication waiver (http://creativecommons.org/publicdomain/zero/1.0/) applies to the data made available in this article, unless otherwise stated. 


\section{Background}

Breast cancer incidence worldwide has risen by $20 \%$ and mortality rates by $14 \%$ since 2008 , with the bulk of this increase sustained by women in low- and middleincome countries (LMICs) due to increasing life expectancy, urbanization, and adoption of Western lifestyles [1]. Although incidence rates have traditionally been low in Asia compared with the U.S. or Europe, due to the sheer population of many Asian countries, the absolute numbers of women with breast cancer in this region has risen dramatically [2].

Several studies have shown that women with breast cancer are at increased risk for osteoporosis and fracture [3-5]. This is largely attributable to the negative impact of breast cancer treatments on skeletal health, which occurs through decreased estrogen exposure [6, 7]. The majority of such studies have been carried out in the U.S. and Europe, where most women are postmenopausal at the time of diagnosis and approximately $75 \%$ of patients have hormone-receptor positive disease [8]. By contrast, a nation-wide epidemiologic study found that the average age at breast cancer diagnosis in China is approximately 10 years earlier than in the West, and only $57.4 \%$ of women with breast cancer had hormonereceptor positive disease [9].

Because of these differences in epidemiology, risk factors for fracture may be significantly different among Chinese breast cancer survivors, and cannot simply be extrapolated from studies in U.S. and European populations. Furthermore, in China, as in many other LMICs, the infrastructure to monitor and manage osteoporosis and fracture is severely limited, with scarce access to dual-energy $x$-ray absorptiometry (DXA) - the goldstandard for bone mineral density assessment-outside of large tertiary care centers in major cities [10].

In order to quantify the scope of this problem and identify potential health services gaps, we designed a cross-sectional study to measure the prevalence of vertebral fractures among a cohort of breast cancer survivors in Beijing, and compared our data with fracture prevalence among community-dwelling healthy women in Beijing selected from a pre-existing cohort called the Peking Vertebral Fracture (PK-VF) Study, and hypothesized that vertebral fracture prevalence would be significantly higher among our cohort of breast cancer survivors.

\section{Methods}

\section{Study design}

We conducted a cross-sectional study comparing vertebral fracture rates and risk factors for fracture among two cohorts in Beijing, China: breast cancer survivors currently receiving care at a single large cancer center, and an age- and body mass index (BMI)-matched group of community-dwelling healthy women recruited as part of a pre-existing epidemiological study of vertebral fracture prevalence in Beijing. This study was reviewed and approved by the institutional review board of the Cancer Hospital, Chinese Academy of Medical Sciences and the human investigations committee of Yale School of Medicine prior to initiation.

\section{Sample \\ Breast cancer survivors cohort}

All breast cancer survivors receiving care at the Cancer Hospital, Chinese Academy of Medical Sciences (CHCAMS) from April 1, 2013 - August 31, 2014 were eligible for this study if they met the following criteria: 1) age 50-70 years, 2) initiated breast cancer treatment at least 5 years prior to enrollment. Exclusion criteria included: 1) initiated breast cancer therapy within last 5 years, 2) history of bone metastases, 2) osteoporosis or osteoporosis therapy prior to breast cancer diagnosis, 3) metabolic or inherited bone disease, 4) corticosteroid or anticonvulsive therapy for $>6$ months or within the last 12 months, 5) conditions leading to secondary osteoporosis (rheumatoid arthritis/connective tissue disease, chronic liver or kidney disease, malabsorption, inflammatory bowel disease, poorly controlled hyperthyroidism). Initially women were recruited by screening the CHCAMS medical database for women diagnosed with breast cancer at CHCAMS prior to January 1, 2008. Potentially eligible women were contacted via telephone and invited to participate in the study. However due to the low success rate of this method during the first month, we switched to a physician referral recruitment approach where all patients presenting for follow up in the breast cancer clinic who met eligibility criteria were referred to the study physician. The study physician met with each potential participant, confirmed eligibility, explained the study purpose, procedures and risks and benefits, and obtained written informed consent.

\section{PK-VF cohort}

Details regarding the recruitment of the PK-VF Study have been described previously [11]. This study recruited a total of 1724 postmenopausal community-dwelling Chinese women in 2008 from seven districts of Beijing, aged 47-108 years. Investigators collected detailed data regarding sociodemographic and clinical characteristics, osteoporosis and fracture-related history and risk factors, thoraco-lumbar x-rays, and serologic samples. For the purposes of this study, we randomly selected an age- and BMI-matched sample of women from the PKVF cohort. 


\section{Measures}

\section{Breast cancer survivors cohort}

Sources of data for each participant included a selfadministered study questionnaire, a medical chart review form completed by the study physician, thoracolumbar x-ray, and fasting serum sample. The study questionnaire consisted of 19 questions that addressed sociodemographic characteristics (age, education level, smoking history, and current alcohol use), history of and risk factors for fracture (height, weight, history of a fall within the last year, parental history of fracture, personal history of fracture, bone mineral density testing or diagnosis of low bone mineral density since breast cancer diagnosis, calcium or vitamin D supplement use), and reproductive health history (age at menarche, parity, age at menopause if applicable, and history of hormone replacement therapy use). Data collected from the medical chart included date of breast cancer diagnosis, stage at diagnosis, pathologic diagnosis, estrogen- and progesterone-receptor status, and HER-2 receptor status. Initial breast cancer treatment history was recorded including type of surgery, and use of radiation therapy, chemotherapy, and/or endocrine therapy [i.e. selective estrogen-receptor modulators (SERMs), aromatase inhibitors (AIs), gonadotropin releasing hormone $(\mathrm{GnRH})$ agonists, ovariectomy]. Data regarding history of recurrence were also obtained.

Thoracic and lumbar lateral spine $\mathrm{x}$-rays were performed to identify prevalent vertebral fractures at the diagnostic imaging department of CHCAMS. For each participant, presence of vertebral fracture was ascertained by two radiologists using the validated Genant semi-quantitative technique [12]. In this method, vertebrae T4-L4 are graded on visual inspection and without direct measurement at normal (grade 0), mildly deformed (grade 1, approximately 20-25\% reduction in anterior, middle, and/or posterior height and a reduction in area of 10-20\%), moderately deformed (grade 2, approximately 25-40 reduction in any height and a reduction in area $20-40 \%$ ), and severely deformed (grade 3, approximately $40 \%$ reduction in any height and area).

Fasting serum samples were collected from each participant and stored at $-80{ }^{\circ} \mathrm{C}$ until batch testing at the end of the study period. We tested 25-hydroxy vitamin $\mathrm{D}$ (25OHD) levels, and the bone formation marker procollagen type $1 \mathrm{~N}$ propeptide (P1NP), and the bone resorption marker serum $\beta$-c-terminal telopeptide of type 1 collagen $(\mathrm{CTx})$ to assess bone metabolism. All biomarkers were assayed at the Di'an laboratory in Beijing, China using an automated Roche electrochemiluminescence immunoassay (cobas e 601, Roche Diagnostics, Basel, Switzerland).

\section{PK-VF cohort}

For each participant, data were extracted from the PKVF Study database regarding participant age, BMI, education level, parity, menstrual history, smoking, alcohol use, history of bilateral ovariectomy, parental history of fracture, personal history of fracture, and calcium supplement use. As part of the PK-VF Study, prevalent vertebral fractures were also ascertained using lateral thoracolumbar x-ray, and evaluated by two experienced radiologists using the Genant semi-quantitative method described above. Serum biomarkers, CTx, P1NP, and $25 \mathrm{OHD}$, were batch tested at the central laboratory of the Department of Endocrinology, Peking Union Medical College Hospital, by an automated Roche electrochemiluminescence immunoassay (Modular Analytics E170; Roche Diagnostics, Basel Switzerland) [11].

\section{Data analysis}

All statistical analyses were performed using Stata Intercooled 13 (StataCorp, College Station, TX). Descriptive statistics were used to report the sociodemographic characteristics, frequency of fracture, and fracturerelated risk factors in the two cohorts. Categorical variables were compared using $X^{2}$ or Fisher's exact test, and continuous variables were compared using the t-test. Univariate logistic regression was further used to calculate the odds of vertebral fracture based upon breast cancer status, age, BMI, level of education, parity, personal history of fracture, calcium supplement use, and 25OHD level. We fit the multivariable model using backward elimination beginning with all variables that were significant $(p$-value $<0.10)$ in the univariate analyses (breast cancer survivor status, age, parity, calcium supplement use) [13]. To obtain a parsimonious model, we removed non-significant variables $(p>0.05)$ one at a time beginning with the least significant; in each step, remaining parameter estimates remained largely unchanged $(<20 \%)$. Among the cohort of breast cancer survivors, we further performed subgroup analyses based upon fracture status using $\chi^{2}$, Fisher's exact test, and the t-test as appropriate.

\section{Results}

Sociodemographic, reproductive and fracture-associated characteristics

In total, 200 survivors of breast cancer were enrolled and 200 matched healthy women were selected from the PK-VF cohort. The mean age of both cohorts was $57.5 \pm$ 4.9 years and over half of women had a BMI above $24 \mathrm{~kg} / \mathrm{m}^{2}$ (Table 1$)$. Women in the breast cancer cohort were more highly educated and smoking and alcohol use were rare among both cohorts. Fewer women with breast cancer self-reported a personal history of fracture (10.5 v. $21 \%, p=0.004)$. Among breast cancer survivors 
Table 1 Sociodemographic, Reproductive, and FractureAssociated Characteristics of BCS and PK-VF Study Cohorts

\begin{tabular}{lll}
\hline Variable & BCS Cohort & PK-VF Cohort \\
\hline Age years & $57.5 \pm 4.9$ & $57.5 \pm 4.9$ \\
BMI kg/m ${ }^{2}$ & $24.8 \pm 3.7$ & $24.7 \pm 3.2$ \\
Education $\geq$ High School & $156 / 200(78)^{\mathrm{c}}$ & $91 / 197(46.1)$ \\
Smoking, Ever & $6 / 200(3)$ & $5 / 200(2.5)$ \\
Current Alcohol Use & $11 / 200(5.5)$ & $8 / 200(4.0)$ \\
Menarche years & $14.4 \pm 2.0^{\mathrm{b}}$ & $15.1 \pm 2.4$ \\
Menopause years & $49.4 \pm 4.0$ & $49.6 \pm 3.5$ \\
Parity & & \\
$\quad 0$ & $2 / 200(1 \%)$ & $2 / 190(1.6)$ \\
$\quad 1$ & $137 / 200(68.5)^{\mathrm{b}}$ & $102 / 190(53.7)$ \\
$\quad \geq 2$ & $61 / 200(30.5)^{\mathrm{a}}$ & $85 / 190(44.7)$ \\
Fall in Past Year & $31 / 198(15.7)$ & - \\
Parental Fracture History & $20 / 188(10.6)$ & $31 / 200(15.5)$ \\
Personal Fracture History & $21 / 200(10.5)^{\mathrm{b}}$ & $42 / 200(21)$ \\
Calcium Supplement Use & $96 / 196(49.0)^{\mathrm{a}}$ & $73 / 198(36.9)$ \\
Vitamin D Supplement Use & $12 / 200(6.0 \%)$ & - \\
CTx ng/mL & $0.458 \pm 0.211$ & $0.441 \pm 0.199$ \\
P1NP ng/mL & $61.1 \pm 30.4$ & $56.3 \pm 24.6$ \\
25OHD ng/mL & $20.3 \pm 7.8^{\mathrm{c}}$ & $13.3 \pm 5.7$ \\
\hline
\end{tabular}

Values for continuous variables are reported as mean $\pm S D$ and for categorical values as $n / N(\%)$

PK-VF Peking Vertebral Fracture study, BCS breast cancer survivors, $C T x$ serum $\beta$-c-terminal telopeptide of type 1 collagen, P1NP pro-collagen type $1 \mathrm{~N}$ propeptide, 25OHD 25-hydroxy vitamin $\mathrm{D}$, $\mathrm{kg}$ kilograms, $m$ meters, $\mathrm{ng}$ nanograms, $m L$ milliliters

$\mathrm{a}<.05$

${ }^{\mathrm{b}}<.01$

$c<.001$

the most commonly reported site of fracture was the wrist $(8 / 21,38.1 \%)$. One patient reported a history of hip fracture and only one self-reported a history of vertebral fracture. Other reported fracture sites included the ankle, lower leg, foot, finger, coccyx, knee, toes, and ribs. Thirty-three percent (6/18) of fractures were reported as low-trauma fractures. More breast cancer survivors reported using calcium supplements $(49$ v. $36.9 \%, p=$ $0.015)$. Only $12 / 200(6 \%)$ of women with breast cancer reported using vitamin D supplements.

\section{Vitamin D status and bone turnover markers}

Mean levels of 25OHD were significantly higher among the breast cancer survivors compared with women in the PK-VF Study $(20.3 \pm 7.8$ v. $13.3 \pm 5.7, p<0.001)$ (Table 1). Even so, 113/196 (58.2\%) of women with breast cancer met criteria for 25OHD deficiency (< $20 \mathrm{ng} / \mathrm{mL}$ ), and 61/196 (35.2\%) met criteria for 25OHD insufficiency (20-29 ng/mL). By contrast, no significant differences in mean levels of bone turnover makers were noted between the two groups. The correlation coefficient between CTx and P1NP was $0.72(p<0.001)$ among breast cancer survivors, and $0.78(\mathrm{p}<0.001)$ among the PK-VF cohort demonstrating appropriate coupling of bone formation and resorption in both groups.

\section{Vertebral fractures}

Table 2 demonstrates the total number of women in the breast cancer survivors cohort $(22 / 200,11 \%)$ and PK-VF cohort $(7 / 200,3.5 \%)$ with prevalent vertebral fractures. Several women had more than one fracture, and the total number of vertebral fractures identified by thoracolumbar $\mathrm{x}$-ray was also significantly greater among breast cancer survivors (47 v. 9 fractures, $\mathrm{p}<0.001)$. Breast cancer survivors were more likely to have multiple fractures as well as higher grade fractures. The odds of having a vertebral fracture among breast cancer survivors compared with their healthy counterparts was 3.41 (95\%CI 1.42-8.17, $p=0.006$ ) (Table 3). In the multivariable model, the odds of vertebral fracture among breast cancer survivors was 4.16 (95\%CI 1.69-10.21, $p=0.002)$ compared with women in the PK-VF cohort.

\section{Characteristics of breast cancer survivors cohort}

Table 4 details the characteristics of the cohort of breast cancer survivors based upon fracture status, including breast cancer-related characteristics. The average duration of breast cancer at the time of enrollment was 6.3 \pm 1.9 years. Approximately $85 \%$ of women were

Table 2 Vertebral Fracture Results for BCS and PK-VF Study Cohorts

\begin{tabular}{lll}
\hline Variable & BCS Cohort & PK-VF Cohort \\
\hline Individuals with VF n/N(\%) & $22 / 200(11 \%)^{\S}$ & $7 / 200(3.5 \%)$ \\
Grade 1, b & 13 & 5 \\
Grade 2 & $12^{\S}$ & 2 \\
Grade 3 & 2 & 0 \\
VFs per Individual median(range) & $1(1-10)$ & $1(1-2)$ \\
Total Number of VF N & $47^{*}$ & 9 \\
Grade 1 & $19^{*}$ & 7 \\
Grade 2 & $25^{*}$ & 2 \\
Grade 3 & 3 & 0 \\
\hline
\end{tabular}

VF vertebral fracture, PK-VF Peking Vertebral Fracture Study, BCS breast cancer survivors

${ }^{*}<.05$

$\S<.01$

$¥<.001$

${ }^{\text {a }}$ Please note that because some individuals have multiple fractures of different grades, the number of individuals with grade 1, grade 2 and grade 3 fractures add up to more than 22

${ }^{b}$ Vertebral Fractures were classified using the Genant Semi-Quantitative technique. In this method, vertebrae T4-L4 are graded as normal (Grade 0), mildly deformed (Grade 1, approximately 20-25\% reduction in anterior, middle, and/or posterior height and a reduction in area of 10-20\%), moderately deformed (Grade 2 , approximately $25-40$ reduction in any height and a reduction in area $20-40 \%$ ), and severely deformed (Grade 3, approximately $40 \%$ reduction in any height and area) ${ }^{13}$ 
Table 3 Logistic Regression Analysis: Odds of Vertebral Fracture among Women in the BCS and PK-VF Study Cohorts, Combined $(N=400)$

\begin{tabular}{|c|c|c|c|c|}
\hline \multirow[t]{2}{*}{ Variable } & \multicolumn{2}{|c|}{ Univariate Model } & \multicolumn{2}{|c|}{ Multivariable Model } \\
\hline & $\overline{O R}$ & $95 \% \mathrm{Cl}$ & $\overline{\mathrm{OR}}$ & $95 \% \mathrm{Cl}$ \\
\hline Breast Cancer Survivor & 3.41 & $1.42-8.17^{c}$ & 4.16 & $1.69-10.21^{c}$ \\
\hline Age years & 1.08 & $1.01-1.17^{c}$ & 1.10 & $1.02-1.20^{b}$ \\
\hline BMl kg/m² & 0.90 & $0.78-1.03$ & - & - \\
\hline Education $\geq$ High School & 0.85 & $0.39-1.83$ & - & - \\
\hline Parity & 1.49 & $0.93-2.37^{\mathrm{a}}$ & - & - \\
\hline Age of Menarche years & 1.08 & $0.92-1.27$ & - & - \\
\hline Personal History of Fracture & 0.60 & $0.18-0.12$ & - & - \\
\hline Calcium Supplement Use & 0.48 & $0.21-1.12^{\mathrm{a}}$ & 0.37 & $0.15-0.89^{b}$ \\
\hline $250 H D$ Level $n g / m L$ & 1.00 & $0.95-1.05$ & - & - \\
\hline
\end{tabular}

Continuous variables: Age, BMI, Parity, Age of Menarche, 25OHD level. Categorical variables: Breast Cancer Survivor (reference: Peking Vertebral Fracture Study participant), Education (reference: $\leq$ middle school), Personal History of Fracture (reference: no history of fracture), Calcium Supplement Use (reference: no supplement use)

$\mathrm{OR}$ odds ratio, $\mathrm{Cl}$ confidence interval, $\mathrm{BMI}$ body mass index, 25OHD 25-hydroxy vitamin D

a $<0.1$

${ }^{\mathrm{b}}<.05$

${ }^{c}<.01$

diagnosed at early stage (stage I or II), and a similar proportion had hormone receptor positive disease on pathology and underwent some form of endocrine therapy during the course of treatment, including SERMS [tamoxifen (56/70) and/or toremifene (21/70)], AIs [Anastrozole (55/90), Letrozole (23/90), and/or Exemestane (13/ 90)] and GnRH agonists [Goserelin (1/2) and Leuprolide $(1 / 2)]$. Sixteen women underwent ovariectomy as part of breast cancer therapy. The average length of treatment with SERMS was $50.5 \pm 21.4$ months, and the average length of treatment with AIs was $58.9 \pm 17.9$ months. Forty-three percent of women were postmenopausal at the time of breast cancer diagnosis, of whom only $29.6 \%$ reported having had a DXA scan since being diagnosed. Of the 90 women who were treated with AI therapy, only $30 \%$ reported having had a DXA scan. Approximately $16 \%$ of breast cancer survivors reported a fall within the last year, and $49 \%$ reported taking a calcium supplement.

Stratified analyses based upon fracture status, showed that women with fracture were older and therefore more likely to be postmenopausal at the time of diagnosis (63.6 v. 40.1\%, $p=0.036$ ), and had a lower level of education (59.1 v. $80.3 \%$ with $\geq$ high school education, $p=$ 0.023). Although our sample was not powered to formally evaluate differences in fracture status based upon treatment class, we observed that vertebral fractures were detected among 7/74 (9.4\%) of women receiving AIs only, 5/54 (9.3\%) receiving SERMs only, 1/16 (6.3\%) who had received both an AI and SERM, and in 3/16 (18.8\%) women who had been treated with ovariectomy. Of note, all three of the ovariectomized women with fractures were premenopausal at the time of diagnosis and had concurrently been treated with a SERM. 8/54 $(14.8 \%)$ of women who received radiation treatment had a vertebral fracture, however 7 of those women were also concurrently treated with some form of endocrine therapy.

Levels of 25OHD, bone turnover markers, and treatment patterns did not vary based upon fracture status.

\section{Discussion}

Our study is the first to directly measure rates of fracture among breast cancer survivors in China compared to age- and BMI-matched healthy women. We found that history of breast cancer was associated with a fourfold increased odds of prevalent vertebral fractures. Furthermore, breast cancer survivors with fractures were more likely to have multiple fractures and higher grade fractures compared to their healthy counterparts. Finally, rates of DXA screening for bone disease were low among women with breast cancer, even among those who were postmenopausal at the time of diagnosis or treated with AIs, factors known to increase risk for fracture.

Rates of fractures have traditionally been higher in Europe and the U.S. compared to China [14]. Therefore, it is consistent that absolute prevalence of fractures in both of our cohorts were lower compared with previous studies among women from Caucasian populations. However, recent studies have also shown that rates of fracture are rapidly increasing in China due to urbanization and adoption of Western lifestyles [15]. In our study, the magnitude of the increased odds for fracture seen among breast cancer survivors is roughly on par with prior findings. Kanis et al., found that prevalence of vertebral fractures among women with softtissue breast cancer recurrence (without skeletal metastases) was six-fold higher compared with healthy controls or women newly diagnosed with breast cancer [4]. However, their study population was acutely ill and mean age was 2 years older than our cohort, whereas our cohort included predominantly recurrence-free breast cancer survivors.

To our knowledge only one prior study has been published from mainland China examining this issue [16]. This study retrospectively compared 70 postmenopausal women with breast cancer receiving AI therapy, with 52 women receiving tamoxifen, and 89 women who were not treated with endocrine therapy at a single institution. The authors found that women on AIs had an increased incidence of fractures $(12.9 \%)$ compared with those not treated with endocrine therapy $(1.1 \%, p=0.001)$. By 
Table 4 Characteristics of BCS Cohort, Overall and by Fracture Status

\begin{tabular}{|c|c|c|c|}
\hline Variable & Overall & No Fracture & Fracture \\
\hline Age years & $57.5 \pm 4.9$ & $57.1 \pm 4.8$ & $60.0 \pm 5.2^{b}$ \\
\hline $\mathrm{BMI} \mathrm{kg} / \mathrm{m}^{2}$ & $24.8 \pm 3.7$ & $24.9 \pm 3.7$ & $23.8 \pm 3.3$ \\
\hline $\begin{array}{l}\text { Education } \geq \text { High } \\
\text { School }\end{array}$ & 156/200 (78) & $143 / 178(80.3)$ & $13 / 22(59.1)^{a}$ \\
\hline Menarche years & $14.4 \pm 2.0$ & $14.5 \pm 1.9$ & $14.5 \pm 2.1$ \\
\hline Menopause years & $49.4 \pm 4.0$ & $49.4 \pm 4.2$ & $49.2 \pm 2.2$ \\
\hline \multicolumn{4}{|l|}{ Parity } \\
\hline 0 & $2 / 200(1)$ & $2 / 178(1.1)$ & $0 / 22(0)$ \\
\hline 1 & $137 / 200(68.5)$ & 126/178 (70.8) & $11 / 22(50)^{\mathrm{a}}$ \\
\hline$\geq 2$ & $61 / 200(30.5)$ & $50 / 178(28.1)$ & $11 / 22(50)^{a}$ \\
\hline Smoking, Ever & $6 / 200(3)$ & $6 / 178(3.4)$ & 0/22 (0) \\
\hline Current Alcohol Use & $11 / 200(5.5)$ & $11 / 178(6.2)$ & $0 / 22(0)$ \\
\hline Fall in Past Year & $31 / 198(15.7)$ & 28/176 (15.9) & 3/22 (13.6) \\
\hline Parental Fracture History & 20/188 (10.6) & 18/167 (10.8) & 2/21 (9.5) \\
\hline Personal Fracture History & $21 / 200(10.5)$ & 19/178 (10.7) & 2/22 (9.1) \\
\hline Calcium Supplement & 96/196 (49) & $88 / 174(50.6)$ & $8 / 22(36.4)$ \\
\hline DXA Since Diagnosis & $54 / 200(27)$ & $44 / 178(24.7)$ & $10 / 22(45.5)^{a}$ \\
\hline $\begin{array}{l}\text { Low BMD Since } \\
\text { Diagnosis }\end{array}$ & 24/195 (12.3) & 22/173 (12.7) & $2 / 22(9.1)$ \\
\hline$C T \times n g / m L$ & $0.458 \pm 0.211$ & $0.457 \pm 0.209$ & $0.463 \pm 0.227$ \\
\hline P1NP ng/mL & $61.1 \pm 30.4$ & $61.3 \pm 30.1$ & $58.9 \pm 33.2$ \\
\hline $250 \mathrm{HD} \mathrm{ng} / \mathrm{mL}$ & $20.3 \pm 7.8$ & $20.5 \pm 7.6$ & $19.0 \pm 9.7$ \\
\hline $\begin{array}{l}\text { Postmenopausal at } \\
\text { Diagnosis }\end{array}$ & $81 / 189$ (42.9) & $67 / 167(40.1)$ & $14 / 22(63.6)^{a}$ \\
\hline $\begin{array}{l}\text { Duration of Breast } \\
\text { Cancer }\end{array}$ & $6.3 \pm 1.9$ & $6.4 \pm 2.0$ & $6.1 \pm 1.6$ \\
\hline \multicolumn{4}{|l|}{$\begin{array}{l}\text { BrCa Stage at } \\
\text { Diagnosis }\end{array}$} \\
\hline 1 & 77/183 (42.1) & 69/162 (42.6) & $8 / 21(38.1)$ \\
\hline$\|$ & 79/183 (43.2) & 69/162 (42.6) & 10/21 (47.6) \\
\hline III & 27/183 (14.8) & 24/162 (14.8) & $3 / 21(14.3)$ \\
\hline \multicolumn{4}{|l|}{$\begin{array}{l}\text { Hormone Receptor } \\
\text { Status }\end{array}$} \\
\hline $\mathrm{ER}+/ \mathrm{PR}+$ & 113/186 (60.1) & $100 / 164(61)$ & 13/22 (59.1) \\
\hline $\mathrm{ER}+/ \mathrm{PR}-$ & 11/186 (5.9) & 10/164 (6.1) & $1 / 22(4.6)$ \\
\hline ER-/PR+ & 33/186 (17.6) & $30 / 164(18.3)$ & $3 / 22(13.6)$ \\
\hline ER-/PR- & 29/186 (15.4) & 24/164 (14.6) & $5 / 22(22.7)$ \\
\hline $\begin{array}{l}\text { HER2 Receptor } \\
\text { Positive }\end{array}$ & 38/183 (20.8) & $35 / 161(21.7)$ & $3 / 22(13.6)$ \\
\hline Surgery & 187/187 (100) & $165 / 165(100)$ & $22 / 22(100)$ \\
\hline $\begin{array}{l}\text { Radiation } \\
\text { Therapy }\end{array}$ & 53/182 (29.1) & $45 / 160(28.1)$ & 8/22 (36.4) \\
\hline Chemotherapy & 153/185 (82.7) & 135/163 (82.8) & 18/22 (81.8) \\
\hline $\begin{array}{l}\text { Endocrine } \\
\text { Therapy }\end{array}$ & 158/189 (84) & $141 / 167(84.4)$ & 17/22 (77.3) \\
\hline SERM & 70/188 (37.2) & 64/166 (38.6) & $6 / 22(27.3)$ \\
\hline $\begin{array}{l}\text { Aromatase } \\
\text { Inhibitor }\end{array}$ & $90 / 189$ (47.6) & 82/167 (49.1) & $8 / 22(36.4)$ \\
\hline
\end{tabular}


Table 4 Characteristics of BCS Cohort, Overall and by Fracture Status (Continued)

\begin{tabular}{llll}
\hline Variable & Overall & No Fracture & Fracture \\
\hline GnRH agonist & $2 / 189(1.1)$ & $2 / 167(1.2)$ & $0 / 22(0)$ \\
Ovariectomy & $16 / 189(8.5)$ & $13 / 167(7.8)$ & $3 / 22(13.6)$ \\
Recurrence & $2 / 185(1.1)$ & $2 / 162(1.2)$ & $0 / 22(0)$ \\
\hline
\end{tabular}

Values for continuous variables are reported as mean $\pm S D$ and for categorical values as $n / N(\%)$

PK-VF Peking Vertebral Fracture Study, BCS breast cancer survivors, DXA dual-energy x-ray absorptiometry, BMD bone mineral density, CTx serum $\beta$-c-terminal telopeptide of type 1 collagen, $P 1 N P$ pro-collagen type $1 \mathrm{~N}$ propeptide, 25OHD 25-hydroxy vitamin D, ER estrogen receptor, PR progesterone receptor, HER2 human epidermal growth factor receptor 2, SERM selective estrogen receptor modulator, GnRH gonadotropin releasing hormone

a $<.05$

$\mathrm{b}^{\mathrm{b}}<.01$

contrast, incidence of fractures among those treated with tamoxifen did not differ $(1.9 \%, p=0.372)$ compared with those not treated with endocrine therapy. Unfortunately, this study is significantly limited by the lack of description regarding how osteoporotic fractures were defined and measured, and sparse risk factor data.

Three studies from Taiwan using retrospective data from their National Insurance Research Database have also examined this issue among ethnically Chinese women with breast cancer. Tzeng et al. reported tamoxifen use was associated with reduced risk of osteoporotic fractures, however did not specify whether women in their cohort were pre- or postmenopausal [17]. Chang et al. studied fracture risk in young breast cancer patients and found that those receiving AIs, radiotherapy, or Herceptin were at increased risk for future fracture [18]. Tsa et al. showed that age-specific hazard ratio for fracture was higher for breast cancer patients $<50$ years of age, both for traumatic and non-traumatic fractures [19]. However, these studies are all based upon insurance claims data and rely on International Conference for the Ninth Revision of the International Classification of Diseases (ICD-9) codes for diagnosis. Validation of the diagnostic codes was not described therefore further studies are needed to confirm these findings in prospective cohorts. Our data provide a meaningful comparison to these studies given the differences in lifestyle, nutrition, medical care and environmental exposures among women in mainland China compared with Taiwan, and provides direct assessment of fracture associated risk factors not available from insurance claims data.

Previous epidemiologic studies from Asian countries have demonstrated that the peak age of breast cancer diagnosis occurs approximately a decade earlier among Asian women compared with their Western counterparts $[20,21]$. The age and menopausal status at diagnosis of our cohort are consistent with data from a nationally representative sample of 4211 Chinese women diagnosed with breast cancer (mean age at diagnosis = $48.7 \pm 10.5$ years, $62.9 \%$ premenopausal). Because the vast majority of studies on this subject have been conducted in Western populations, the long-term impact of breast cancer treatment on bone health among Asian women remains unknown. Furthermore, less is known about the long-term risk for fracture among premenopausal women and guidelines cannot simply be extrapolated from studies for postmenopausal women with breast cancer.

As a population, Chinese women undergoing treatment for breast cancer will become vulnerable to fracture at a younger age relative to their Western counterparts, during a period when comparatively little attention is typically given to fracture risk reduction. Furthermore, even when increased fracture risk is identified, management algorithms are less straightforward for premenopausal women compared with postmenopausal women. In 2012, Hadji et al. published a review of the complexities of cancer treatment-induced bone loss (CTIBL) among premenopausal women, and proposed a framework with which to approach this problem including indications for DXA screening and calcium and vitamin D supplementation [22]. However, in China, as in other rapidly modernizing Asian countries, lack of access to DXA and osteoporosis specialists is common, and presents a barrier to comprehensive fracture risk assessment. In 2013, there were only an estimated 0.0046 DXA machines per 10,000 individuals in China, which is far below the density ( 0.11 per 10,000 population) recommended by the International Osteoporosis Foundation [10]. The lack of DXA access at even the highesttier cancer centers, including CHCAMS, also suggests a fundamental lack of recognition and prioritization at the health systems level regarding the long-term impact of CTIBL on health outcomes.

From a practice perspective, our study underscores the importance of awareness of fracture risk associated with breast cancer therapies both on the part of the provider and patient. Although fracture rates were relatively high, due to the asymptomatic nature of most vertebral fractures, the vast majority of women and their providers were not aware of these fractures. While guidelines exist in China for screening and management of CTIBL, it is apparent that gaps remain in terms of uptake of these recommendations in practice. Indeed, among breast 
cancer survivors with a prevalent vertebral fracture, less than $50 \%$ had obtained a DXA scan. While $49 \%$ of breast cancer survivors were on calcium supplements, only $9 \%$ were taking vitamin D supplements. Given age and postmenopausal status are known risk factors for fractures, it is not surprising that overall, more fractures occurred among women who were postmenopausal at the time of diagnosis. However, it is important to note that $8 / 108$ $(7.4 \%)$ of breast cancer survivors who were premenopausal at the time of diagnosis were also found to have vertebral fractures, which underscores the excess risk for fractures in this population. While studies have demonstrated bisphosphonates may be effective for mitigating CTIBL, such studies have not been powered to measure impact on fracture rates [23-25].

Our study has several limitations that warrant mention. First, the PK-VF cohort has a few key differences compared with the breast cancer survivors cohort. It was enrolled 5 years earlier than the breast cancer survivors cohort, and carried out by a separate group of investigators and radiologists. However, the principal investigator of the PK-VF was a collaborator on this study and extensive care was taken to parallel the methodologies of the two studies to ensure comparability of findings. Second, although all breast cancer survivors were $>50$ years of age, at baseline $6.5 \%$ of women in the breast cancer survivors cohort were not yet in menopause. This group also had higher education levels, earlier menarche, lower parity rates, lower personal fracture history, higher rates of calcium supplement use, and higher baseline 25OHD levels. During the design of our study, to avoid potential bias due to overmatching, we did not choose to match our controls based upon each of these factors and instead, took them into account in our regression analyses. As these characteristics would have been expected to be associated with lower risk of fracture, if anything, our findings would underestimate the risk of fracture among Chinese breast cancer survivors. Third, given the cross-sectional design of our study, our data do not allow for calculation of incidence rates of vertebral fracture over time, nor change in laboratory parameters. Our study was also not powered to look for subgroup analyses based upon treatment regimen, therefore we are unable to provide formal comparisons of fracture rates by type of treatment or duration of those treatments. Finally, our study population was limited to breast cancer survivors presenting for routine follow up at a major cancer hospital in Beijing, and therefore may not generalizable to all Chinese women with breast cancer, nor to other regions of China.

\section{Conclusion}

In summary, our study found breast cancer survivors in China have a four-fold increased odds of prevalent vertebral fracture compared with age- and BMI-matched healthy women. However, rates of screening for osteoporosis and fracture risk by DXA were low reflecting a lack of standardization of care regarding screening, prevention and treatment of this problem. Future longitudinal studies are needed to elucidate the fracture risk specific to women who are premenopausal at the time of diagnosis and how risk relates to treatment regimens. Finally, infrastructure limitations such as lack of access to DXA imaging at cancer hospitals remain important barriers to timely intervention.

\section{Abbreviations \\ 25OHD: 25-hydroxy vitamin D; Al: Aromatase inhibitors; BCS: Breast cancer survivors; BMI: Body mass index; CHCAMS: Cancer Hospital, Chinese Academy of Medical Sciences; CTIBL: Cancer treatment-induced bone loss; CTx: $\beta-c-$ terminal telopeptide of type 1 collagen; DXA: Dual-energy $x$-ray absorpti- ometry; ER: Estrogen receptor; GnRH: Gonadotropin releasing hormone; HER2: Human epidermal growth factor receptor 2; ICD-9: International Conference for the Ninth Revision of the International Classification of Diseases; LMIC: Low- and middle-income countries; P1NP: Pro-collagen type $1 \mathrm{~N}$ propeptide; PK-VF: Peking Vertebral Fracture; PR: Progesterone receptor; SERM: Selective estrogen-receptor modulator}

\section{Acknowledgements}

Our sincere appreciation to the women at the Cancer Hospital, Chinese Academy of Medical Sciences who took part in this study and to the participants of the Peking Vertebral Fracture Study. Special thanks to Dr. Steven Cummings and Dr. Elizabeth Bradley for their advice and insights during development of this study. Thanks also to Jianyun Zhao, Priya Sivasubramaniam, Qian Zhang, and Shaoming Wang for their valuable contributions to data collection, laboratory testing, data management and organization.

\section{Funding}

The project and Dr. Evelyn Hsieh were supported by the NIH Research Training Grant \# R25 TW009340 funded by the Fogarty International Center, the NIH Office of the Director Office of Research on Women's Health, the Office of AIDS Research and the National Cancer Institute. Dr. Liana Fraenkel is supported by NIAMS K24 AR060231-01. The funding body had no role in the design of the study and collection, analysis, and interpretation of data and in writing the manuscript.

\section{Availability of data and materials}

The datasets used and/or analyzed during the current study are available from the corresponding author on reasonable request.

\section{Prior presentation of data}

These data have been presented at the European Calcified Tissue Society Meeting Annual Meeting (May 14-17, 2016. Rome, Italy). Preliminary data from this study have previously been presented at the American Society for Bone Mineral Research (September 12-15, 2014. Houston, TX), and the International Conference on Osteoporosis and Bone Mineral Research (October 16-19, 2014. Xiamen, China)

\section{Authors' contributions}

This study was designed by EH, WX, LF, KLI, JSS, CZ, YQ, and ZP. Data collection and quality control for the Breast Cancer Cohort was carried out by $E H, Q W, N X, R Z$, and ZP. Radiographs were read by RZ and L. WX provided access and permission to use data from the PKVF Study. Data analysis was performed by EH. Data was interpreted by EH, QW, LF, KI, ZP. Manuscript organization and writing was undertaken by $\mathrm{EH}$ with detailed input from QW, LF, KLI, ZP. All authors participated in the manuscript review and approved the final version of the text as submitted to BMC Cancer $(E H$, QW, RZ, XN, WX, LF, KLI, JL, JSS, CZ, YQ, PZ). 


\section{Ethics approval and consent to participate}

This study was reviewed and approved by the institutional review board of the Cancer Hospital, Chinese Academy of Medical Sciences (approval reference number: 12-133/667) and the Human Investigation Committee of Yale School of Medicine (approval reference number: 1,301,011,316) prior to initiation. All procedures performed in this study involving human participants were performed in accordance with the ethical standards laid down in the 1964 Declaration of Helsinki and its later amendments. Written informed consent was obtained from all individual participants included in the study.

\section{Consent for publication}

Not applicable

\section{Competing interests}

The authors declare that they have no competing interests.

\section{Publisher's Note}

Springer Nature remains neutral with regard to jurisdictional claims in published maps and institutional affiliations.

\section{Author details}

${ }^{1}$ Section of Rheumatology, Yale School of Medicine, New Haven, CT, USA 2Department of Ultrasound, National Cancer Center/Cancer Hospital, Chinese Academy of Medical Sciences \& Peking Union Medical College, Beijing, China. ${ }^{3}$ Department of Diagnostic Radiology, National Cancer Center/Cancer Hospital, Chinese Academy of Medical Sciences \& Peking Union Medical College, Beijing, China. ${ }^{4}$ Department of Cancer Epidemiology, National Cancer Center/Cancer Hospital, Chinese Academy of Medical Sciences \& Peking Union Medical College, Beijing, China. ${ }^{5}$ Department of Endocrinology, Key Laboratory of Endocrinology, Peking Union Medical College Hospital, Beijing, China. ${ }^{6}$ Section of Endocrinology, Yale School of Medicine, New Haven, CT, USA. ${ }^{7}$ Department of Epidemiology, UNC Gillings School of Global Public Health, Chapel Hill, NC, USA. ${ }^{8}$ Department of Medical Oncology, National Cancer Center/Cancer Hospital, Chinese Academy of Medical Sciences \& Peking Union Medical College, Beijing, China.

Received: 6 March 2017 Accepted: 22 January 2018

Published online: 30 January 2018

\section{References}

1. Ferlay J, Soerjomataram I, Dikshit R, Eser S, Mathers C, Rebelo M, et al. Cancer incidence and mortality worldwide: sources, methods and major patterns in GLOBOCAN2012. Int J Cancer. 2015;136(5):E359-86

2. $\quad$ Song $\mathrm{QK}$, Wang $\mathrm{XL}$, Zhou XN, Yang HB, Li YC, Wu JP, et al. Breast cancer challenges and screening in China: lessons from current registry data and population screening studies. Oncologist. 2015;20:773-9.

3. Chen Z, Maricic M, Bassford TL, Pettinger M, Ritenbaugh C, Lopez AM, et al. Fracture risk among breast cancer survivors: results from the Women's health initiative observational study. Arch Intern Med. 2005;165(5):552-8.

4. Kanis JA, McCloskey EV, Powles T, Paterson AH, Ashley S, Spector T, et al. A high incidence of vertebral fracture in women with breast cancer. $\mathrm{Br}$ Cancer. 1999;79(7/8):1179-81.

5. Melton $\amalg$ III, Hartmann LC, Achenbach SJ, Atkinson EJ, Therneau TM, Khosla S. Fracture risk in women with breast cancer: a population-based study. J Bone Miner Res. 2012:27(5):1196-205.

6. Body JJ. Increased fracture rate in women in breast cancer: a review of the hidden risk. BMC Cancer. 2011:11:384

7. Guise TA. Bone loss and fracture risk associated with cancer therapy Oncologist. 2016:11:1121-31.

8. Chu KC, Anderson WF, Fritz A, Ries LA, Brawley OW. Cancer frequency distributions of breast cancer characteristics classified by estrogen receptor and progesterone receptor status for eight racial/ethnic groups. Cancer. 2001:92:37-45

9. Li J, Zhang BN, Fan JH, Pang Y, Zhang P, Wang SL, et al. A nation-wide multicenter 10-year (1999-2008) retrospective clinical epidemiological study of female breast cancer in China. BMC Cancer. 2011:11:364.

10. Mithal A, Ebeling P. The Asia-Pacific regional audit: epidemiology, costs and burden of osteoporosis in Asia. Hong Kong: International Osteoporosis Foundation; 2013. https://www.iofbonehealth.org/sites/default/files/media/
PDFs/Regional\%20Audits/2013-Asia_Pacific_Audit_0_0.pdf. Accessed 20 Feb 2017

11. Zhao J, Xia WB, Nie M, Zheng X, Wang Q, Wang X, et al. The levels of bone turnover markers in Chinese postmenopausal women: Peking vertebral fracture study. Menopause. 2011;18(11):1237-43.

12. Genant HK, Wu CY, Van Kuijk C, Nevitt MC. Vertebral Fracture Assessment Using a Semiquantitative Technique. J Bone Miner Res. 1993;8(9):1137-48.

13. Sauerbrei W, Royston $P$, Binder H. Selection of important variables and determination of functional form for continuous predictors in multivariable model building. Stat Med. 2007;26(30):5512-28.

14. Lau EMC, Cooper C, Fung H, Lam D, Tsang KK. Hip fracture in Hong Kong over the last decade - a comparison with the UK. J Public Health Med. 1999:21(3):249-50.

15. Xia WB, He SL, Xu L, Liu AM, Jiang Y, Li M, et al. Rapidly increasing rates of hip fracture in Beijing, China. J Bone Miner Res. 2012;27(1):125-9.

16. Xu L, Wang J, Xue DD, He W. Aromatase inhibitors associated musculoskeletal disorders and bone fractures in postmenopausal breast cancer patients: a result from Chinese population. Med Oncol. 2014;31:128

17. Tzeng HE, Muo CH, Chen HT, Hwang WL, Hsu HC, Tsai CH. Tamoxifen use reduces the risk of osteoporotic fractures in women with breast cancer in Asia: a nationwide population-based cohort study. BMC Musculoskelet Disord. 2015:16:123.

18. Chang CH, Chen SJ, Liu CY. Fracture risk and adjuvant therapies in young breast cancer patients: a population-based study. PLoS One. 2015;10(6): e0130725

19. Tsa $\mathrm{CH}$, Muo $\mathrm{CH}$, Tzeng HE, Tang $\mathrm{CH}$, Hsu HC, Sung FC. Fracture in Asian women with breast cancer occurs at younger age. PLoS One. 2013;8(9): e75109.

20. Chen C, Sun S, Yuan JP, Wang YH, Cao TZ, Zheng HM, et al. Characteristics of breast cancer in Central China, literature review and comparison with the USA. Breast. 2016; https://doi.org/10.1016/j.breast.2016.01.004. [epub ahead of print]

21. Sivasubramaniam PG, Zhang BL, Zhang Q, Smith JS, Zhang B, Tang ZH, et al. Breast cancer disparities: a multicenter comparison of tumor diagnosis, characteristics, and surgical treatment in China and the U.S. Oncologist. 2015;20(9):1044-50.

22. Hadji P, Gnant M, Body JJ, Bundred NJ, Brufsky A, Coleman RE, et al. Cancer treatment-induced bone loss in premenopausal women: a need for therapeutic intervention? Cancer Treat Rev. 2012;38:798-806.

23. Gnant M, Mlineritsch B, Luschin-Ebengreuth $G$, Kainberger F, Kässmann H, Piswanger-Sölkner JC, et al. Adjuvant endocrine therapy plus zoledronic acid in premenopausal women with early-stage breast cancer: 5-year follow-up of the ABCSG-12 bone-mineral density substudy. Lancet Oncol. 2008;9(9):840-9.

24. Brufsky AM, Harker WG, Beck JT, Bosserman L, Vogel C, Seidler C, et al. Final 5-year results of Z-FAST trial: adjuvant zoledronic acid maintains bone mass in postmenopausal breast cancer patients receiving letrozole. Cancer. 2012; 118(5):1192-201.

25. Coleman R, de Boer R, Eidtmann H, Llombart A, Davidson N, Neven P, et al. Zoledronic acid (zoledronate) for postmenopausal women with early breast cancer receiving adjuvant letrozole (ZO-FAST study): final 60-month results. Ann Oncol. 2013;24(2):398-405.

\section{Submit your next manuscript to BioMed Central and we will help you at every step:}

- We accept pre-submission inquiries

- Our selector tool helps you to find the most relevant journal

- We provide round the clock customer support

- Convenient online submission

- Thorough peer review

- Inclusion in PubMed and all major indexing services

- Maximum visibility for your research

Submit your manuscript at www.biomedcentral.com/submit
Biomed Central 